\title{
Opportunity Advantage between Income Distributions
}

\author{
Carmen Herrero \\ FAE, University of Alicante \& Ivie
}

Antonio Villar

Pablo de Olavide University

Carmen Herrero:

Postal address: Department of Economics, University of Alicante, 03080 Alicante, Spain.

Email: cherreroblanco@gmail.com

Antonio Villar (corresponding author)

Postal address: Department of Economics, Universidad Pablo de Olavide, 41013 Seville, Spain.

Email: avillar@upo.es 
Keywords: domination probabilities, income distribution, opportunity advantage, ranking distributions, veil of ignorance.

\begin{abstract}
This paper seeks to compare income distributions from the opportunity advantage viewpoint. That is a measure of how likely it is for a representative individual of one society to receive a higher income than a representative individual of another. Opportunity advantage tries to measure the income opportunities a society offers to an individual, relative to other societies, evaluated from the "veil of ignorance" viewpoint. We show that this notion can be precisely formalised and results in a complete and cardinal income distribution evaluation. We also present an empirical illustration of the gender differences in wage distributions in Spain after the financial crisis. The results show that this criterion offers new insights into the evaluation of differences in opportunity.
\end{abstract}

JEL classification numbers: D31, D63, I30 


\section{Introduction}

Comparing income distributions is a basic tool of welfare economics and is probably one of the most serious steps to move beyond the GDP when assessing the economic situation of different societies. Those comparisons can be performed from different perspectives, depending on the aspect one wants to focus on, and involve diverse measurement protocols. There is a well-established literature on the comparison of income distributions from the point of view of inequality, poverty, polarization, or discrimination (e.g., Chakravarty, 2009; Villar, 2017).

We propose to address here the evaluation of income distributions focusing on the opportunities they offer to a representative member of the society in terms of the probability of getting a higher income. We consider that the income distribution of Society A exhibits higher opportunity than that of Society B, when it is more likely that a member of $\mathrm{A}$, drawn at random, obtains a higher income than a member of B, also chosen randomly. As those probabilities depend on how many individuals are above each income level within each society, this comparison can be interpreted as applying the utilitarian-flavoured principle of more income for more people. ${ }^{1}$

The idea of comparing pairs of income distributions from the point of view of the probability of getting higher incomes has already appeared in the literature dealing with the analysis of discrimination. Gastwirth (1975) introduced this principle to study wage discrimination between men and women. To do so, he calculated the "probability that a randomly chosen woman earns at least as much as

\footnotetext{
${ }^{1}$ Bentham speaks of "the greatest happiness of the greatest number" in the Preface of his 1776 work "A Fragment on Government" (Bentham 1891, facsimile edition, p. 93).
} 
a randomly selected man" (Cf. p. 32). The deviation of this probability from the value 0.5 was a measure of discrimination. He applied this evaluation protocol to the USA's white population at different points in time and in different industries, using continuous distribution functions. In a similar vein, Lieberson (1976) uses this notion to measure segregation between two societies, A and B. He builds an indicator in which he compares the probability that an agent from Society A achieves higher outcomes than one from Society B and the complementary event. Lieberson uses discrete distributions and measures discrimination by the absolute value of the difference of those probabilities.

Both the Gastwirth (1975) and Lieberson (1976) evaluation protocols can be understood as generalizations of the first-order stochastic dominance criterion, in the sense that whenever a distribution A first-order stochastically dominates another one, B, then it has a Garstwirth index over 0.5 , or the difference between the dominance probabilities is positive. Contrary to the first-order stochastic dominance criterion, that only yields a partial ordering, these two measures are complete; that is, they can be used to rank any pair of income distributions. Le Breton, Michelangeli \& Peluso (2012) provided a new indicator of "discrimination patterns" referring to distributions that are compared indirectly through a pair-wise evaluation of each one relative to a common reference distribution. They analysed first- and second-order discrimination curves in a vein similar to first- and secondorder stochastic dominance and link the Gastwirth discrimination index to the second-order discrimination curve. Salas, Bishop \& Zeager (2018) introduced a new definition of second-order discrimination without the resource to a common reference distribution in the same line of work. In all these cases, only partial orderings are obtained. 
This paper presents a protocol to evaluate income distributions based on the idea of opportunity advantage. This notion tries to capture the chances of obtaining a higher income in a society, relative to the others, much in the spirit of Gastwirth (1975) and Lieberson (1976). The opportunity advantage of a Society A relative to a Society B is directly proportional to the probability that a representative agent of Society A obtains a higher income than a representative agent of Society B. We call this the proportionality principle.

The opportunity advantage protocol differs substantially in scope, both conceptually and operationally, from all those contributions mentioned above. Conceptually, opportunity advantage differs from inequality, segregation, discrimination, polarization, or poverty measures. Making the evaluation of income distributions proportional to the chances of having higher incomes implies that we move away from the realm of the measures that satisfy Dalton's principle of transfers (opportunity advantage does not satisfy second-order stochastic dominance). Yet our criterion can be interpreted in terms of inequality of opportunity when applied to population subgroups that only differ in their external circumstances (see below).

The operational difference in scope is also relevant. The opportunity advantage is a complete, cardinal, and transitive criterion applicable to any number of societies. It provides an evaluation of the income distribution of each society under consideration, relative to all others. Why does this make a difference? On the one hand, note that all those measures based on stochastic dominance comparisons provide only partial orderings. On the other hand, as in Gastwirth (1975) and Lieberson (1976), discrimination measures are complete but can only be applied to pairs of societies, as binary comparisons are not transitive. 
In the case of those criteria that only permit comparisons of pairs, an extension to more than two societies can be obtained by making indirect comparisons. The income distribution of each society is evaluated relative to a reference distribution, which is the same for all societies under consideration. This is also the case of inequality measures, which take the perfect egalitarian allocation as the "natural" reference distribution and then define inequality as some type of distance between the actual distribution and the reference one.

The opportunity advantage takes a different venue to yield a transitive evaluation. Rather than using a common reference distribution, it computes the distance between each distribution and all others in a precise and systematic way. This is obtained by applying a consistency criterion, which allows the proportionality principle to be extended from two to any number of distributions. Consistency here is defined by requiring that the evaluation protocol, for the case of more than two societies, should coincide with that obtained by applying the binary proportionality principle to each society relative to the fictitious one that would result from merging all others.

The paper is organized as follows. Section 2 presents the evaluation protocol by focusing on the comparison of two societies and then extending that criterion consistently to any finite number. Two intuitive properties suffice to characterize the evaluation formula. The discussion of the scope and properties of this protocol is presented in Section 3. We shall see that the evaluation can be regarded as a cardinal, complete, and transitive extension of the first-order stochastic dominance criterion. We shall also discuss how to use this evaluation protocol to deal with the analysis of inequality of opportunity and poverty. Section 4 concludes by providing 
an empirical illustration in which we measure the opportunity advantage of the wage distributions between men and women in Spain after the financial crisis.

\section{The opportunity advantage approach to income distributions}

This paper presents here a way of transforming the idea of comparing income distributions, in terms of the chances of getting higher incomes, into a precise evaluation formula: the opportunity advantage. This formula is derived from two intuitive properties. When comparing two income distributions, the first one says that the evaluation attached to each one is proportional to the likelihood of getting higher incomes. The second is a consistency requirement that extends this principle to any number of income distributions. We shall derive the evaluation formula in this section and discuss its meaning and properties in the next one.

Consider a collection of $M=\{1,2, \ldots, m\}$ societies, whose income distributions are to be compared. ${ }^{2}$ For each society $k$, let $f_{k}$ denote the associated probability density function; that is, for each $y \in \mathbb{R}_{+}, f_{k}(y)$ represents the (relative) mass of population in society $k$ with income $y$. We shall write $\left(f_{i}\right)_{i=1}^{m}=\left\{f_{1}, \ldots, f_{m}\right\}$. By construction, $\int_{0}^{+\infty} f_{k}(y) d y=1$ for all $k$.

The key ingredient for the evaluation is the probability $q_{k j}$ that an individual from Population $k$ obtains a higher income than an individual from Population $j$, for

\footnotetext{
2 The formulation presented below is compatible with both continuous and discrete income distributions. Hence, the integrals that appear are to be regarded as Riemann's integrals that correspond to sums when distributions are discrete. We also assume that incomes belong to $\mathbb{R}_{+}$, for the sake of simplicity in exposition, even though no such a requirement is necessary (i.e. we can take the whole real line as the income range).
} 
any pair $k, j \in M$. We shall refer to this variable as the domination probability of $k$ over $j$. To obtain such a probability, we calculate how often an individual from Society $k$ has an income higher than an individual from Society $j$, both drawn at random. ${ }^{3}$ To calculate these domination probabilities, it is convenient to consider, for any density $f_{k}$, the cumulative distribution function:

$$
F_{k}(y)=\int_{0}^{y} f_{k}(t) d t
$$

The probability that an agent drawn at random from $f_{k}$ has an income greater than $y$ is given by $1-F_{k}(y)$, while $f_{j}(y)$ reflects the probability of an agent in $f_{j}$ having income $y$. Then, the domination probability of $k$ over $j$, denoted by $q_{k j}$, is given by:

$$
q_{k j}=\int_{0}^{+\infty} f_{j}(y)\left[1-F_{k}(y)\right] d y=1-\int_{0}^{+\infty} f_{j}(y) F_{k}(y) d y
$$

Similarly, $q_{j k}=1-\int_{0}^{+\infty} f_{k}(y) F_{j}(y) d y$.

Since $q_{k j}+q_{j k}=1$, by construction, we can write:

$$
q_{k j}=\int_{0}^{+\infty} f_{k}(y) F_{j}(y) d y ; \quad q_{j k}=\int_{0}^{+\infty} f_{j}(y) F_{k}(y) d y
$$

An evaluation problem, or merely a problem involving $m$ income distributions, refers to the comparison of the corresponding vector of densities, $\left(f_{i}\right)_{i=1}^{m}$. Let $\mathbf{P}=\left\{\left(f_{i}\right)_{i=1}^{m}, \forall m \in \mathbb{N}, m \geq 2\right\}$ denote the set of all those problems for any $m \geq 2$. An evaluation function is a mapping $\varphi$ that, for each problem $\left(f_{i}\right)_{i=1}^{m} \in$ $\mathbf{P}$, associates a vector $\varphi\left(\left(f_{i}\right)_{i=1}^{m}\right) \in \mathbb{R}_{+}^{m}$. We aim to identify an evaluation function that provides a sound measure of the opportunity advantage, understood as the

\footnotetext{
${ }^{3}$ In the discrete case, when both agents have the same income level, we split the probability mass evenly.
} 
relative likelihood of getting higher incomes.

First consider the simplest case in which we compare the income distributions of two societies, $M=\{k, j\}$. Our first property, proportionality, makes it explicit that we prioritize that income distribution that gives "more income to more people" by evaluating both distributions proportionally to their corresponding domination probabilities. That is,

Proportionality: For each problem $\left(f_{k}, f_{j}\right) \in \mathbf{P}$,

$$
\frac{\varphi_{k}\left(f_{k}, f_{j}\right)}{\varphi_{j}\left(f_{k}, f_{j}\right)}=\frac{q_{k j}}{q_{j k}}
$$

This is an elementary evaluation principle that provides a sensible measure of the income opportunity that a particular society offers to its members, relative to the other. The proportionality principle fully determines the evaluation, for the twosociety case, except for the choice of units, as there is a degree of freedom (multiplying $\varphi_{k}, \varphi_{j}$ by a scalar $\lambda>0$ does not change Equation [1]).

By writing Equation [1] as:

$$
q_{j k} \varphi_{k}\left(f_{k}, f_{j}\right)=q_{k j} \varphi_{j}\left(f_{k}, f_{j}\right)
$$

We can interpret $q_{k j} \varphi_{j}\left(f_{k}, f_{j}\right)$ as the advantage of $k$ over $j$ (how many times $k$ is better than $j$, times the valuation of $j$ ) and, similarly, $q_{j k} \varphi_{k}\left(f_{k}, f_{j}\right)$ as the advantage of $j$ over $k$. The proportionality requirement amounts to equating both advantages.

Let us see what this property tells us about the evaluation of the income distributions in the following example, where we consider three societies with the following densities (without loss of generality we can assume that all have the same per capita income): 


$$
\begin{gathered}
f_{A}(y)=\left\{\begin{array}{l}
1 \text { if } y=2 \\
0 \text { otherwise }
\end{array}\right. \\
f_{B}(y)=\left\{\begin{array}{c}
0.4 \text { if } y=0.5 \\
0.6 \text { if } y=3 \\
0 \text { otherwise }
\end{array}\right. \\
f_{C}(y)=\left\{\begin{array}{c}
0.3 \text { if } y=0 \\
0.2 \text { if } y=0.5 \\
0.1 \text { if } y=3 \\
0.4 \text { if } y=4 \\
0 \text { otherwise }
\end{array}\right.
\end{gathered}
$$

We here have $q_{A B}=0.4, q_{B A}=0.6$. By normalizing the values so that the mean is equal to 1 , we obtain: $\varphi_{A}\left(f_{A}, f_{B}\right)=0.8 ; \varphi_{B}\left(f_{A}, f_{B}\right)=1.2$. From the opportunity advantage viewpoint, income distribution $B$ is better than income distribution $A$ (and we have a measure of how much better: one third better). This shows that this criterion does not satisfy Dalton's principle of transfers. The intuition of why Society B gets a higher evaluation is that $60 \%$ of its members obtain more than the average income.

As regards distribution $C, q_{A C}=q_{C A}=0.5$ and thus, $\varphi_{A}\left(f_{A}, f_{C}\right)=$ $1 ; \varphi_{C}\left(f_{A}, f_{C}\right)=1$. Hence, income distributions $\boldsymbol{y}_{A}$ and $\boldsymbol{y}_{C}$ are indifferent from the opportunity advantage viewpoint. The comparison of distributions $\boldsymbol{y}_{B}$ and $\boldsymbol{y}_{C}$ yields $q_{B C}=0.49 ; q_{C B}=0.51$, and therefore, $\varphi_{B}\left(f_{B}, f_{C}\right)=0.98 ; \varphi_{C}\left(f_{B}, f_{C}\right)=1.02$. That is, $\boldsymbol{y}_{B}$ is better than $\boldsymbol{y}_{A}$ (as shown above), which is indifferent to $\boldsymbol{y}_{C}$, which happens to be better than $\boldsymbol{y}_{B}$. A cycle that makes it impossible to rank those three distributions, $\boldsymbol{y}_{A}, \boldsymbol{y}_{B}, \boldsymbol{y}_{C}$ in terms of pair-wise comparisons.

The previous example illustrates that pair-wise comparisons in terms of the proportionality principle cannot be directly extended to more than two societies because the order induced is not transitive.

How can we extend the proportionality principle to the case of more than 
two distributions in a systematic way? A standard procedure in many contexts requires some form of consistency when moving from two to many (e.g., Thomson 2011). This is a regularity requirement on the behaviour of a rule when changing the number of agents involved. It usually permits an intuitive principle defined for the two-agent case to be applied to a general scenario. Here we apply this notion by associating a set of $m$ derived problems involving two distributions each, which contains information on the whole, to any problem involving $m>2$ distributions.

The most natural way of constructing pair-wise problems while keeping track of all distributions' information is by taking expectations. That is, for each problem $\left(f_{i}\right)_{i=1}^{m} \in \mathbf{P}$, we construct $m$ two-dimensional problems in which we confront each distribution with the expectation of all others in the problem.

To present this requirement, let us define the overall advantage of society $k$ as the expected advantage of $k$ over all other societies. That is,

$$
A_{k}\left(\left(f_{i}\right)_{i=1}^{m}, \varphi\right)=\frac{1}{m-1} \sum_{j \neq k} q_{k j} \varphi_{j}\left(\left(f_{i}\right)_{i=1}^{m}\right)
$$

Trivially, when $m=2, A_{k}\left(\left(f_{k}, f_{j}\right), \varphi\right)=q_{k j} \varphi_{j}\left(f_{k}, f_{j}\right)$ and we are back to the definition above.

For a problem $\left(f_{i}\right)_{i=1}^{m} \in \mathbf{P}$, with $m>2$, let $\left(f_{k}, f_{-k}\right)$ denote the twodimensional evaluation problem consisting of Society $k$ and the society that would result from merging all other societies into a single one, denoted by $M_{-k}$. That is, $f_{-k}=E\left[\left(f_{j}\right)_{j \neq k}\right]$, where $E$ is the mathematical expectation. Let $\varphi_{k}\left(f_{k}, f_{-k}\right)$, $\varphi_{-k}\left(f_{k}, f_{-k}\right)$ stand for the evaluation of societies $k$ and $M_{-k}$, respectively, in problem $\left(f_{k}, f_{-k}\right)$. The property of consistency introduces the principle that this merging process keeps the ratio between the evaluations of Society $k$ in both scenarios equal to the corresponding overall advantages. Formally: 
Consistency: For each problem $\left(f_{i}\right)_{i=1}^{m} \in \mathbf{P}, m>2$,

$$
\frac{\varphi_{k}\left(\left(f_{i}\right)_{i=1}^{m}\right)}{\varphi_{k}\left(f_{k}, f_{-k}\right)}=\frac{A_{k}\left(\left(f_{i}\right)_{i=1}^{m}, \varphi\right)}{A_{k}\left(\left(f_{k}, f_{-k}\right), \varphi\right)}
$$

That is, the ratio between the value attached to income distribution $k$ in the original problem $\left(f_{i}\right)_{i=1}^{m}$ and that in the modified problem $\left(f_{k}, f_{-k}\right)$ corresponds, precisely, to the ratio of its overall advantages in both scenarios.

The following result shows that the evaluation formula deriving from those two properties is one in which the value attached to income distribution $k$ is directly proportional to its overall advantage and inversely proportional to the average probability that some other distribution dominates $k$. In other words, the extension to the general case of the proportionality principle, as expressed in Equation [1'], amounts to taking expectations.

Proposition: An evaluation function $\varphi$, defined in the family of problems $\mathbf{P}$, satisfies proportionality and consistency if and only if, for each $m \in \mathbb{N}, m \geq 2$, it attaches to each income distribution in problem $\left(f_{i}\right)_{i=1}^{m} \in \mathbf{P}$, the value $\varphi_{k}\left(\left(f_{i}\right)_{i=1}^{m}\right) \in \mathbb{R}_{+}^{m}$ given by:

$$
\varphi_{k}\left(\left(f_{i}\right)_{i=1}^{m}\right)=\frac{\sum_{j \neq k} q_{k j} \varphi_{j}\left(\left(f_{i}\right)_{i=1}^{m}\right)}{\sum_{j \neq k} q_{j k}} \quad k, j=1,2, \ldots, m
$$

$\underline{\text { Proof }}$

We first show that any evaluation function that satisfies proportionality and consistency corresponds to that formula. We shall then show that such a function is well defined; that is, that for each problem $\left(f_{i}\right)_{i=1}^{m} \in \mathbf{P}$ we can ensure the existence of those values $\varphi_{1}\left(\left(f_{i}\right)_{i=1}^{m}\right), \varphi_{2}\left(\left(f_{i}\right)_{i=1}^{m}\right), \ldots, \varphi_{m}\left(\left(f_{i}\right)_{i=1}^{m}\right)$.

(i) Let $\varphi$ be an evaluation function that satisfies those two properties. For a 
problem involving two income distributions, $\left(f_{1}, f_{2}\right)$, proportionality yields the desired result. Now take the case of a problem $\left(f_{i}\right)_{i=1}^{m}$, for $m>2$, and any $k$ consider the problem $\left(f_{k}, f_{-k}\right)$ associated with it. By proportionality,

$$
\frac{\varphi_{k}\left(f_{k}, f_{-k}\right)}{\varphi_{-k}\left(f_{k}, f_{-k}\right)}=\frac{\frac{1}{m-1} \sum_{j \neq i} q_{k j}}{\frac{1}{m-1} \sum_{i \neq j} q_{j k}}
$$

By consistency, and bearing in mind the definition of overall advantage, we can write:

$$
\frac{\varphi_{k}\left(\left(f_{i}\right)_{i=1}^{m}\right)}{\varphi_{k}\left(f_{k}, f_{-k}\right)}=\frac{A_{k}\left(\left(f_{i}\right)_{i=1}^{m}, \varphi\right)}{A_{k}\left(\left(f_{k}, f_{-k}\right), \varphi\right)} \Rightarrow \varphi_{k}\left(\left(f_{i}\right)_{i=1}^{m}\right)=\varphi_{k}\left(f_{k}, f_{-k}\right) \frac{\sum_{j \neq k} q_{k j} \varphi_{j}\left(\left(f_{i}\right)_{i=1}^{m}\right)}{\left(\sum_{j \neq k} q_{k j}\right) \varphi_{k}\left(f_{k}, f_{-k}\right)}
$$

That is,

$$
\varphi_{k}\left(\left(f_{i}\right)_{i=1}^{m}\right)=\frac{\sum_{j \neq k} q_{k j} \varphi_{j}\left(\left(f_{i}\right)_{i=1}^{m}\right)}{\left(\sum_{j \neq k} q_{k j}\right)}
$$

A mapping that satisfies proportionality and consistency must have the format described in Equation [2]. As this expression has a degree of freedom, we can normalise the units so that the mean of the evaluation equals 1 .

(ii) Let us now show that this mapping is well defined; that is, for each problem, there is a vector $\mathbf{v} \in \mathbb{R}_{+}^{m}$ with this property. Let $\Delta=\left\{\mathbf{x} \in \mathbb{R}_{+}^{m} / \sum_{i=1}^{m} x_{i}=\right.$ $m\}$ and consider the function $h: \Delta \rightarrow \mathbb{R}^{m}$ given by:

$$
h_{i}(\mathbf{x})=x_{i}-\frac{1}{m-1}\left(x_{i} \sum_{j \neq i} q_{i j}-\sum_{j \neq i} q_{j i} x_{j}\right)
$$

As $\sum_{j \neq i} q_{i j} \leq m-1$, we have:

$$
h_{i}(\mathbf{x}) \geq x_{i}-x_{i}+\frac{1}{m-1} \sum_{j \neq i} q_{j i} x_{j} \geq 0
$$

Moreover,

$$
\sum_{i=1}^{m} h_{i}(\mathbf{x})=m-\frac{1}{m-1}\left(\sum_{i=1}^{m} x_{i} \sum_{j \neq i} q_{i j}-\sum_{i=1}^{m} \sum_{j \neq i} q_{j i} x_{j}\right)
$$


Note that, by construction, $\sum_{i=1}^{m} x_{i} \sum_{j \neq i} q_{i j}=\sum_{i=1}^{m} \sum_{j \neq i} q_{j i} x_{j}$, which means that $\sum_{i=1}^{m} h_{i}(\mathbf{x})=m$. That is function $h$ maps $\Delta$ into itself. As $h$ is a continuous function and $\Delta$ is a non-empty compact convex set, Brouwer's Theorem (e.g., Border, 1985) ensures the existence of a fixpoint, $\mathbf{v}=h(\mathbf{v})$. That is,

$$
v_{i}=\frac{\sum_{j \neq i} q_{i j} v_{j}}{\sum_{j \neq i} q_{j i}}, \quad i=1,2, \ldots, m
$$

\section{Q.e.d.}

The evaluation of the three distributions $A, B, C$ above, under proportionality and consistency, normalising the values so that the mean equals 1 , is given by: $\varphi_{A}\left(f_{A}, f_{B}, f_{C}\right)=0.868, \varphi_{B}\left(f_{A}, f_{B}, f_{C}\right)=1.118$, and $\varphi_{C}\left(f_{A}, f_{B}, f_{C}\right)=1.014$

\section{Discussion}

Opportunity advantage is a criterion to evaluate income distributions based on the likelihood of getting higher incomes. It captures the relative opportunities that different societies' income distributions offer to their citizens from a "veil of ignorance" perspective. Consequently, $\varphi_{k}\left(\left(f_{i}\right)_{i=1}^{m}\right)>\varphi_{j}\left(\left(f_{i}\right)_{i=1}^{m}\right)$ does not mean that distribution $f_{k}$ is "better than" distribution $f_{j}$ from a normative viewpoint. It merely says that it gives relatively more chance of getting a higher income. Our contribution consists of precisely formalising that measurement rod. This is intrinsically a relative measure in which each distribution is evaluated with respect to the others. It is not meaningful otherwise (it is not even defined for the case of a single distribution). 
The evaluation formula derives from two key properties. The first, proportionality, clarifies that we place more value on income distribution providing higher incomes to more people when comparing two societies. The second one, consistency, ensures that this pair-wise principle can be appropriately extended to more than two societies. The evaluation formula is not trivial, but it is very intuitive. As indicated in Equation [2], each society's evaluation consists of the ratio of its overall advantage over the average probability of being dominated by some other distribution.

Calculating the opportunity advantage requires solving an $m$-dimensional homogeneous system of simultaneous equations, and thus the solution has a degree of freedom. This type of equation system corresponds to a familiar process that appears in different contexts, such as the Google page-rank algorithm (Brin \& Page, 1998), the more sophisticated evaluation of the intellectual influence of scientific journals (e.g., Palacios-Huerta \& Volij 2004, West, Bergstrom \& Bergstrom 2010) and the balanced worth, used to make comparisons in terms of categorical variables (Herrero \& Villar 2018). ${ }^{4}$

We have normalized the evaluation by setting $\sum_{i} \varphi_{i}\left(\left(f_{i}\right)_{i=1}^{m}\right)=m$ in the numerical examples presented in Section 2. This way of closing the formula allows those distributions that exhibit evaluations above or below the mean to be directly identified. This is just a particular way of fixing the units of measurement. Other options might be more convenient in some cases (e.g., setting the highest value to 100 so that the remaining values provide the percentage of the top performer as in the empirical illustration below).

\footnotetext{
${ }^{4}$ Interestingly, when distributions are discrete, we can recur to a free-access algorithm that directly provides the valuation of the distributions: https://web2011.ivie.es/balanced-worth/.
} 
We now discuss some of the critical features of this evaluation protocol.

\subsection{Stochastic dominance and other properties}

It is easy to check that when an income distribution shifts to higher values while the rest remains unchanged, the corresponding valuation increases. This monotonicity property is related to first-order stochastic dominance. If an income distribution first-order stochastically dominates another, its evaluation shall be higher from the opportunity advantage viewpoint. More formally, if $f_{k}, f_{j}$ are two densities that are not identical and such that $F_{k}(y) \leq F_{j}(y), \forall y \in \mathbb{R}_{+}$, then, $\varphi_{k}\left(\left(f_{i}\right)_{i=1}^{m}\right)>\varphi_{j}\left(\left(f_{i}\right)_{i=1}^{m}\right)$. From this perspective, the opportunity advantage criterion can be regarded as an extension of the stochastic dominance principle in three ways. First, it ensures that we can compare any distribution pair (i.e., it is a complete criterion rather than a partial one). Second, the comparison can be extended transitively to any number of distributions (i.e., it permits going beyond pair-wise comparisons). And third, it provides a quantitative measure of the difference between distributions (a cardinal evaluation rather than a ranking).

From a social choice perspective, this evaluation protocol satisfies universal domain (there is no restriction on income distributions) and the Pareto principle (an implication of the monotonicity property mentioned above). It is straightforward that it also satisfies the properties of anonymity, symmetry, and scale-independence. That is because the evaluation depends on the distributions but not on the "names" of the agents (any reallocation of agents to incomes does not affect the domination probabilities). Moreover, if we multiply the incomes of all societies by a scalar $\lambda>0$, 
then the corresponding evaluations do not change. Trivially, if two societies exhibit identical income distributions, then their evaluations are also identical.

This evaluation protocol fails to satisfy both independence of irrelevant alternatives and second-order stochastic dominance, as shown in the numerical example presented above. On the one hand, the evaluation of $\mathbf{y}_{A}$ and $\mathbf{y}_{B}$ changed when we considered a third alternative $\mathbf{y}_{C}$; on the other hand, $\mathbf{y}_{A}$ was not ranked first, so that it does not correspond to any measure that complies with Dalton's principle of transfers. ${ }^{5}$

\subsection{Population subgroups}

The model presented in Section 2 provides an evaluation of the income opportunities between different societies under the implicit assumption of homogeneous agents. It can also be applied to analyse income opportunities within a given society with a heterogeneous population. The idea is that we can classify people in this society into several population subgroups, according to some demographic or sociological characteristics (e.g., age, gender, level of studies, the region of residence). In this context, we can apply the opportunity advantage approach to evaluate the impact of those characteristics on income distributions by treating each population subgroup as a different society.

Note that the existence of opportunity differences among population subgroups does not imply an intrinsic fairness component. Opportunity advantage may be regarded as a measure of unfairness in some cases, though, when we

\footnotetext{
${ }^{5}$ Note that, as this criterion satisfies universal domain, the Pareto principle and anonymity, it cannot satisfy independence of irrelevant alternatives, by Arrow's impossibility theorem. This is also the case when make indirect comparisons through a given reference distribution: changing the reference distribution shall alter the relative values of any pair of distributions.
} 
compare income distributions between men and women with similar characteristics other than gender, say. Yet, in other cases, it simply becomes a descriptive indicator of the different opportunities derived from some conditioning variable, as when we compare earnings distributions of different employed workers depending on their university degrees (which might be regarded as a guide to decide on which studies to follow).

This normative edge opens the question of how opportunity advantage relates to equality ofopportunity, one of the most relevant approaches to distributive justice (see Fleurbaey 2008, Roemer \& Trannoy 2015, 2016 for a discussion and detailed references). The best-known version of the inequality of opportunity principle among economists is probably based on Roemer's work $(1996,1998)$. He argued that an outcome distribution can be regarded as the result of two different effects: effort and opportunity. Effort has to do with responsibility and involves people's free choices on a "level playing field." Opportunity refers to the agents' external circumstances, including genes, race, gender, family socioeconomic and cultural background, and other aspects for which agents cannot be held responsible. A fair society should take into consideration the agents' differences in opportunity but not those differences derived from autonomous personal decisions.

The idea that outcome differences due to external circumstances are unfair is a powerful one. Opportunity advantage can be used to measure the degree of unfairness when population subgroups are defined, à la Roemer, by those people who share similar circumstances. The differences between the income distributions of those population subgroups reflect individuals' different opportunities depending on their external circumstances. Our approach's implicit assumption is that each type's income distribution is a sufficient estimate of its opportunity. 
Consequently, a fair society is one in which the opportunity advantages of the different population subgroups are all alike and the chances open to individuals do not depend on their external circumstances. This idea agrees with the ex-ante approach to equality of opportunity, defended in Ramos \& Van de gaer $(2015,2020)$. This value judgment can be regarded as an instance of a basic non-discrimination principle by which we try to ensure that any new member of society shall have access to its average chances, no matter which social group they end up in.

\subsection{Poverty}

Let us see how we can apply this evaluation protocol to poverty measurement. Consider a collection of $m$ societies with income distributions $\left(f_{i}\right)_{i=1}^{m}$. Let $z$ denote the poverty line that applies to all societies ${ }^{6}$ and define:

$$
f^{z}(y)=\left\{\begin{array}{l}
f(y) \text { if } y<z \\
1-\int_{y=0}^{z} f(y) d y \text { for } y=z \\
0 \text { otherwise }
\end{array}\right.
$$

That is, $f^{z}$ is a truncated density that treats all incomes above the poverty line as having a single income value. This amounts to assuming the property of focus that establishes that we are not concerned about how incomes are distributed above the poverty line (see, for instance, Chakravarty 2009).

The opportunity advantage applied to the problem $\left(f_{i}^{z}\right)_{i=1}^{m}$ provides a relative poverty evaluation based on the proportion of people below the poverty line and the income distribution among the poor in different societies. Clearly, $\varphi_{k}\left(\left(f_{i}^{Z}\right)_{i=1}^{m}\right)>\varphi_{j}\left(\left(f_{i}^{Z}\right)_{i=1}^{m}\right)$ means that Society $k$ gives better opportunities to the

\footnotetext{
${ }^{6}$ Each society may well have its own poverty line (e.g. $60 \%$ of its median), but we adopt here a single poverty line for the sake of simplicity in exposition.
} 
poor than Society $j$. To make of this indicator a more conventional "poverty measure," we can set $\max _{k} \varphi_{k}\left(\left(f_{i}^{z}\right)_{i=1}^{m}\right)=1$ and then let $\pi_{j}\left(\left(f_{i}^{z}\right)_{i=1}^{m}\right)=1-$ $\varphi_{j}\left(\left(f_{i}^{z}\right)_{i=1}^{m}\right)$. Note, though, that since this is a relative criterion, poverty zero here does not mean that there are no poor people in society but that the society with $\pi_{j}()=$.0 is the one with better chances for the poor. ${ }^{7}$

\section{An empirical illustration: wage differences by gender in Spain}

We now consider an illustration of this methodology when analysing wage differentials between men and women in Spain. The data come from the Spanish Structure of Earnings Survey 2014 (Encuesta de Estructura Salarial, INE 2017). This is the last wave with available microdata and describes the situation at the beginning of the recovery after the financial crisis (when the gender difference was smaller than before the crisis). This survey is performed every four years in all Member States of the European Union using a common methodology. Data on wages are collected individually, together with several environmental variables. This study enables relationships to be analysed between salary and variables, in particular, the level of education attained, that can contribute to determining its amount. The key aspect of this survey, from our viewpoint, is that it provides information not only on average wages but also on the wage distribution. The survey involves some 228,000 Spanish workers grouped according to their main characteristics (occupation, type

\footnotetext{
${ }^{7}$ A See also the "growth incidence curve" proposed by Ravallion \& Chen (2003) to assess whether growth is pro-poor by considering how the growth rate for a given quantile varies across quantiles ranked by income.
} 
of contract, seniority, gender, education, etc.). The study contains the elevation factors required to obtain information on the active population in Spain. ${ }^{8}$

We focus on the distribution of earnings in terms of (gross) hourly wages for men and women, grouped into three levels of educational attainment: Low Education (compulsory education or less), Medium Education (upper secondary studies), and High Education (tertiary studies). Analysing hourly wages, rather than yearly earnings, is crucial because it cancels the effect of part-time contracts, which in Spain are mostly held by women. ${ }^{9}$ The hourly wage range was restricted to be between $€ 2$ or less and $€ 62$ or more, ${ }^{10}$ using discrete data with $€ 2$ intervals (i.e., gross hourly wages between $€ 2$ and 4 , between $€ 4$ and $€ 6$, etc.). We provide primary data in the Appendix for replication purposes.

In the last quarter of 2014, when the survey was conducted, Spain's activity rate was about $66 \%$ for men and $54 \%$ for women, with unemployment rates of $22.6 \%$ and $24.9 \%$, respectively. It is worth noting that women had higher educational levels than men: $33.43 \%$ of the employed women had low education ( $42.54 \%$ of the men), $23.72 \%$ medium education ( $21.63 \%$ of the men), and $42.85 \%$ high education (35.83\% of the men).

\footnotetext{
${ }^{8}$ Regarding the population, the survey applies to all employed workers registered in the social security system during a given month in the reference year, excluding those high managers whose main earnings are not salaries. Regarding economic sectors, the data refer to the three main economic sectors: industry, construction and services. No data are provided regarding agri-fishing activities and only part of those employed in the public sector are considered.

${ }^{9}$ The differences between yearly labour earnings between men and women are much higher, as they combine differences in reward per hour worked and differences in the number of hours worked during the year. The gender differences in the number of hours worked per period is linked to the extent of part-time jobs, $3 / 4$ of which are held by women, According to the data of the INE (2017) the differences between yearly earnings between men and women are about $30 \%$ whereas the differences between hourly wages are less than half this figure.

${ }^{10}$ This is for simplicity in exposition, avoiding outliers (even though it creates an anomalous picture in the right tail of the men with higher education). This simplification does not affect the evaluation.
} 
Figures 1, 2, and 3 provide the distribution densities of hourly wages for men and women by level of studies. A simple inspection of those pictures clarifies that chances are different for men and women conditional on the level of studies. The reasons are mostly to do with the asymmetric distribution of employment between men and women by sectors and occupations, on the one hand, and to the effects of the complements to the base salary, on the other (e.g., Sanz de Galdeano \& Terskaya, 2020). The strong skewness in the distributions over the selected range is also noticeable. The median wage for workers with low education is about € 9 for men and $€ 7$ for women; for those with secondary studies, €11 for men and €8 for women; and for those with tertiary studies, €17 for men and €13 for women.

[ Figures 1,2,3 about here]

The evaluation protocol presented in Section 2 allows the size of those differences between men and women on the whole distribution of wages (the gender advantage opportunity) to be estimated. Along with the differences due to educational attainments within "Men" and within "Women". Tables 1 and 2 contain that information.

Table 1 provides the measure of opportunity advantage between men and women for each educational level. We set the values of men at each educational level to 100 so that women's chances appear as a percentage of that value. Three immediate messages derive from those data. First, there is substantial gender bias, even when considering hourly wages (women have between 39\% and 47\% fewer chances than men). Second, higher education reduces discrimination with respect to Low and Medium education. And third, the highest difference is not between those with primary studies but between those with secondary studies.

[Table 1 about here] 
Using the data regarding the shares of women in different education levels, we can obtain an index of equality of opportunity as the weighted average of the values in Table 1 . That is,

$$
E_{\text {OppW }}=0.3342 \times 54.82+0.2372 \times 52.95+0.4285 \times 60.6=56.85
$$

Women, therefore, exhibit about $57 \%$ of the opportunities for men.

Table 2 describes how educational levels affect the chances within "Men" and within "Women". Here we set the value of those with higher education to 100 and compare the performance of the other levels in that respect. Differences in opportunity advantage are much larger than those due to gender (and have a different meaning). The opportunities for those with lower education are less than one third than for those with higher education. That figure is about one half for those with secondary studies. We also observe that relative differences are higher among women.

[Table 2 about here]

We have mentioned that the differences in opportunity due to gender and educational attainments may have a different meaning. Differences in opportunity advantage due to gender can be regarded as a measure of inequality of opportunity, under the assumption that a similar education means a similar level of effort. That is, precisely, why differences due to education have a different meaning as the choice of education level is, at least partly, a personal decision. ${ }^{11}$

Finally, Table 3 presents an evaluation of all six population subgroups' opportunity advantage, relative to men with tertiary education. The differences increase as both gender and level of studies have now been taken into account. Women with low education have less than one-fifth of the chances of men with high education (while men with low education are about one-third). Women with medium education are slightly above one-quarter and those with high education slightly below $60 \%$. Men with medium education account for about one half of those with high education.

\footnotetext{
${ }^{11}$ Note that, even in a country where the cost of university studies is very small, social differences affect the aspirations of the young and hence led to differences in participation and success in tertiary studies.
} 
[Table 3 about here]

The application of the opportunity advantage principle to the evaluation of the wage distribution in Spain has shown that there are very large differences by gender and even larger by educational levels. The distributional bias between men and woman, for all educational levels, is much higher than that corresponding to the median values (see Table 4 in the Appendix). The median of the women relative to the men for low, medium and high education levels are $74 \%, 70.1 \%$ and $75.5 \%$, respectively, values well above those in Table 1 . In the same vein, the median of low and medium educated men (resp. women) relative to the high educated are $53.3 \%$ and $66.8 \%$ (resp. $52.2 \%$ and $62 \%$ ). Here again we find that these differences are much smaller than those shown in Table 2. Finally, setting the median of highly educated men equal to 100 , we find that de median of the other groups are as follows: men with low education 53.3\%, men with medium education $66.8 \%$, women with low education $39.4 \%$, women with medium education $46.8 \%$, and women with high education 75.5\%. Once more those values report differences much smaller than those shown in Table 3.

Let us conclude by pointing out that the reason why opportunity advantage is more sensitive than the median to the differences between social groups is because it is an evaluation procedure that takes into account the disparities along the whole distributions, rather than at some singular points. 


\section{Acknowledgments:}

We thank two anonymous referees and the Associate Editor K. Decancq for very helpful comments that led to a substantial rewriting and improvement of the original paper. We have also benefited from the discussion with Joan Esteban. The

first author acknowledges financial support from the Spanish Ministerio de Economía, Industria y Competitividad under projects EC02015-65820P and PID2019-107081GB-I00, the Generalitat Valenciana under PROMETEO 2109/037, and IMeRA. The second author acknowledges financial support from the Spanish Ministerio de Economía, Industria y Competitividad under project EC02015-65408R (MINECO/FEDER). 


\section{REFERENCES}

Bentham, J.: A Fragment on Government, Clarendon Press, London (1891). Facsimile of the 1776 edition.

Border, K.C.: Fixed point theorems with applications to economics and game theory, Cambridge University Press, Cambridge (1985)

Brin, S., Page, L.: The anatomy of a large-scale hypertextual web search engine. Computer Networks and ISDN Systems, 30: 107-117 (1998)

Chakravarty, S.R.: Inequality, polarization, and poverty. Advances in distributional analysis. Springer, New York (2009)

Fleurbaey, M.: Fairness, Responsibility, and Welfare. Oxford University Press, Oxford (2008)

Gastwirth, J.L.: Measures of earnings differentials. The American Statistician, 29(1): $32-35$ (1975)

Herrero, C. , Villar, A. : The Balanced Worth: A procedure to evaluate performance in terms of ordered attributes, Social Indicators Research, 140: 1279-1300 (2018)

INE: Encuesta de Estructura Salarial (EES). Metodología, Instituto Nacional de Estadística, Madrid. Microdata available at: https://www.ine.es/dyngs/INEbase/es/operacion.htm?c=Estadistica C\&ci $\underline{\mathrm{d}=1254736177025 \& \text { menu }=\text { resultados } \& \operatorname{secc}=1254736195110 \& \mathrm{idp}=12547}$ $\underline{359765}$ (2017)

Le Breton, M., Michelangeli, A., Peluso, E.: A stochastic dominance approach to the 
measurement of discrimination. Journal of Economic Theory, 147: 1342$1350(2012)$

Lieberson, S.: Rank-sum comparisons between groups. Sociological Methodology, 7: 276-291 (1976)

Palacios-Huerta, I., Volij, 0.: The Measurement of Intellectual Influence, Econometrica, 72: 963-977 (2004)

Ramos, X., Van de gaer, D.: Empirical Approaches to Inequality of Opportunity: Principles, Measures and Evidence, Journal of Economic Surveys, 30(5): 855883 (2015)

Ramos, X., Van de gaer, D.: Is inequality of opportunity robust to the measurement approach?, Review of Income and Wealth, doi:10.1111/roiw.12448 (2020)

Ravallion, M., Chen, S.: Measuring pro-poor growth, Economics Letters, 78: 93-99 (2003)

Roemer, J.: Theories of Distributive Justice, Harvard University Press, Cambridge: Massachusetts (1996)

Roemer, J.: Equality of Opportunity, Harvard University Press, Cambridge: Massachusetts (1998)

Roemer, J. E., Trannoy, A.: Equality of Opportunities. Chapter 4 in AB Atkinson and F. Bourguignon (eds) : Handbook of Income Distribution, pp. 217-300, North Holland, Amsterdam. (2015)

Roemer, J.E., Trannoy, A.: Equality of opportunity: Theory and measurement. Journal of Economic Literature, 54(4), 1288-1332 (2016)

Salas, R., Bishop, J., Zeager, L.: Second-Order Discrimination and Generalized Lorenz dominance, Review of Income and Wealth, 64(3): 563-575 (2018)

Sanz de Galdeano, A., Terskaya, A.: The labour market in Spain 2002-2018“, IZA 
World of Labour (2020)

Thomson, W.: Consistency and its converse: an introduction, Review of Economic Design, 15: 257-291 (2011)

Villar, A.: Lectures on Inequality, Poverty, and Welfare, Springer-Verlag, Berlin (2017)

West, J.D., Bergstrom. T.C., Bergstrom, C.T.: The Eigenfactor ${ }^{\mathrm{TM}}$ Metrics: A network approach to assessing scholarly journals, College and Research Libraries, 71: $236-244(2010)$ 
APPENDIX: Primary data for the empirical application

[Table 4 about here] 


\section{FIGURES}

Figure 1: Distribution of hourly wages for men and women with Low

\section{Education}

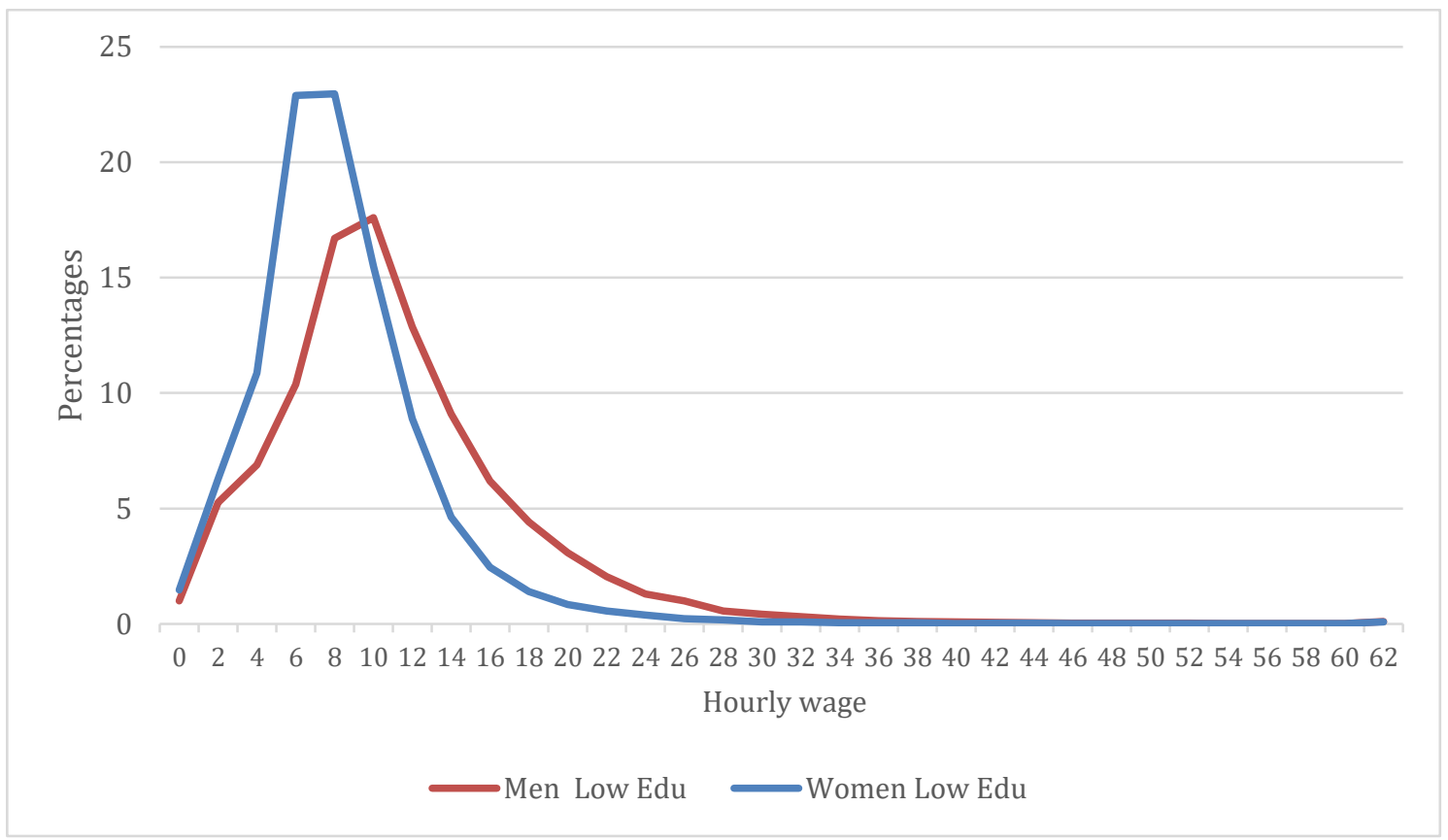

Source: INE (2017)

Figure 2: Distribution of hourly wages for men and women with Medium

\section{Education}

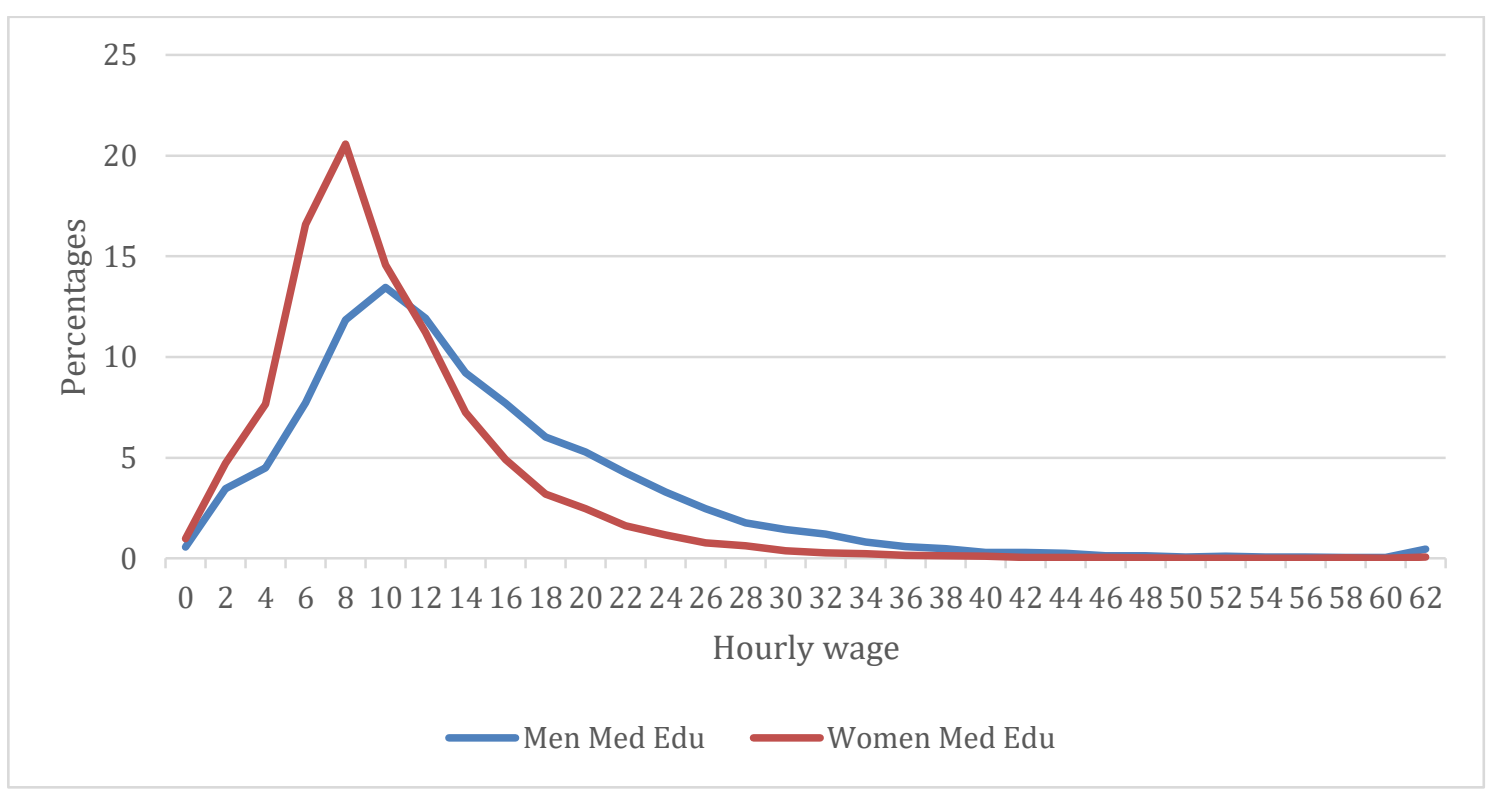


Source: INE (2017)

Figure 3: Distribution of hourly wages for men and women with High

\section{Education}

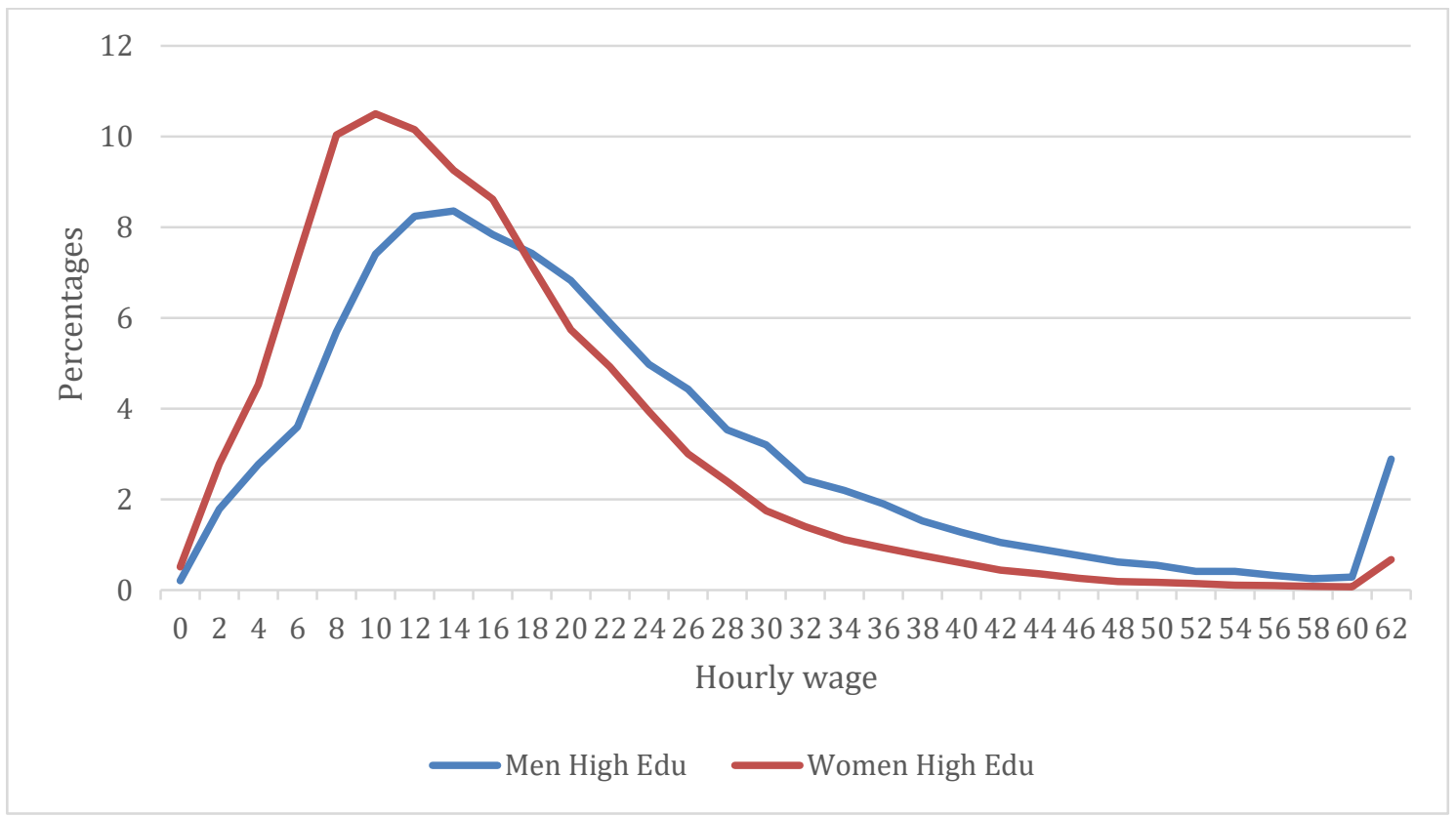

Source: INE (2017) 


\section{TABLES}

Table 1: Differences in opportunity advantage for men and women by educational attainment

\begin{tabular}{|l|r|r|r|}
\hline & Low Edu & Med Edu & High Edu \\
\hline Men & 100 & 100 & 100 \\
\hline Women & 54.82 & 52.95 & 60.6 \\
\hline
\end{tabular}

Table 2: Differences in opportunity advantage by educational levels within men and women

\begin{tabular}{|l|r|r|}
\hline Men & Women \\
\hline Low Education & 31.29 & 30.38 \\
\hline Migh Education & 100 & 100 \\
\hline
\end{tabular}

Table 3: Differences in opportunity advantage (men and women and level of studies)

\begin{tabular}{|l|l|l|}
\hline Low Education & 31.64 & 17.62 \\
\hline Medium Education & 50.28 & 26.48 \\
\hline High Education & 100 & 59.57 \\
\hline
\end{tabular}

Table 4: Distribution of gross hourly wages between men and women by the level of studies (\%)

\begin{tabular}{|c|c|c|c|c|c|c|}
\hline $\begin{array}{l}\text { Hourly wage } \\
(€)\end{array}$ & $\begin{array}{l}\text { Men Low } \\
\text { Edu }\end{array}$ & $\begin{array}{l}\text { Women Low } \\
\text { Edu }\end{array}$ & $\begin{array}{l}\text { Men Med } \\
\text { Edu }\end{array}$ & $\begin{array}{l}\text { Women } \\
\text { Med Edu }\end{array}$ & $\begin{array}{l}\text { Men High } \\
\text { Edu }\end{array}$ & $\begin{array}{l}\text { Women High } \\
\text { Edu }\end{array}$ \\
\hline 0 & 1.001548 & 1.470736 & 0.5627505 & 0.9846415 & 0.2070827 & 0.5084746 \\
\hline 2 & 5.248819 & 6.270682 & 3.457447 & 4.725337 & 1.784634 & 2.784876 \\
\hline 4 & 6.89128 & 10.86005 & 4.49815 & 7.665128 & 2.773512 & 4.526728 \\
\hline 6 & 10.382 & 22.89 & 7.71662 & 16.58815 & 3.594862 & 7.298566 \\
\hline 8 & 16.70489 & 22.9769 & 11.85245 & 20.58325 & 5.698264 & 10.03129 \\
\hline 10 & 17.59472 & 15.49955 & 13.4559 & 14.57646 & 7.406115 & 10.50065 \\
\hline 12 & 12.87509 & 8.891266 & 11.9334 & 11.23151 & 8.243752 & 10.15124 \\
\hline 14 & 9.098214 & 4.62279 & 9.208295 & 7.250542 & 8.357764 & 9.25163 \\
\hline 16 & 6.193528 & 2.450112 & 7.701202 & 4.918496 & 7.834241 & 8.612777 \\
\hline 18 & 4.407989 & 1.397199 & 6.036077 & 3.203618 & 7.427056 & 7.152543 \\
\hline 20 & 3.088924 & 0.8323027 & 5.280604 & 2.478093 & 6.824422 & 5.741851 \\
\hline
\end{tabular}




\begin{tabular}{|r|r|r|r|r|r|r|}
\hline $\mathbf{2 2}$ & 2.042296 & 0.5615537 & 4.251465 & 1.630076 & 5.88906 & 4.920469 \\
\hline $\mathbf{2 4}$ & 1.291625 & 0.3843968 & 3.299414 & 1.17309 & 4.979292 & 3.937418 \\
\hline $\mathbf{2 6}$ & 0.9917485 & 0.2172678 & 2.462997 & 0.7726373 & 4.4325 & 3.006519 \\
\hline $\mathbf{2 8}$ & 0.5566335 & 0.1671291 & 1.757632 & 0.6360124 & 3.534366 & 2.393742 \\
\hline $\mathbf{3 0}$ & 0.4155152 & 0.0869071 & 1.437712 & 0.3863187 & 3.199311 & 1.752282 \\
\hline $\mathbf{3 2}$ & 0.3096764 & 0.0735368 & 1.198736 & 0.2732498 & 2.433803 & 1.397653 \\
\hline $\mathbf{3 4}$ & 0.2038376 & 0.0501387 & 0.8209991 & 0.230849 & 2.198799 & 1.116037 \\
\hline $\mathbf{3 6}$ & 0.1371984 & 0.040111 & 0.5974407 & 0.1554697 & 1.905626 & 0.9335071 \\
\hline $\mathbf{3 8}$ & 0.1038788 & 0.040111 & 0.4856614 & 0.1224913 & 1.528689 & 0.7666232 \\
\hline $\mathbf{4 0}$ & 0.0803591 & 0.0167129 & 0.2967931 & 0.1036465 & 1.277398 & 0.6023468 \\
\hline $\mathbf{4 2}$ & 0.0568393 & 0.0334258 & 0.3006476 & 0.047112 & 1.047047 & 0.4380704 \\
\hline $\mathbf{4 4}$ & 0.0411595 & 0.0267406 & 0.2466852 & 0.0424008 & 0.9051142 & 0.357236 \\
\hline $\mathbf{4 6}$ & 0.0333196 & 0.0200555 & 0.1233426 & 0.0518232 & 0.7608544 & 0.2633637 \\
\hline $\mathbf{5 0}$ & 0.0274397 & 0.0066852 & 0.1310515 & 0.0329784 & 0.6189213 & 0.1929596 \\
\hline $\mathbf{5 2}$ & 0.0215598 & 0.0100277 & 0.0655257 & 0.0047112 & 0.5444646 & 0.1747067 \\
\hline $\mathbf{5 4}$ & 0.0176398 & 0.0033426 & 0.0809436 & 0.0094224 & 0.4141654 & 0.1095176 \\
\hline $\mathbf{5 6}$ & 0.0156798 & 0.0033426 & 0.0693802 & 0.0047112 & 0.3280748 & 0.1016949 \\
\hline $\mathbf{5 8}$ & 0.0156798 & 0.0066852 & 0.0539624 & 0.023556 & 0.2559449 & 0.0782269 \\
\hline $\mathbf{6 0}$ & 0.0078399 & 0.0066852 & 0.0462535 & 0.0094224 & 0.2885197 & 0.0756193 \\
\hline $\mathbf{6 2}$ & 0.1077987 & 0.080222 & 0.4548258 & 0.0659568 & 2.892177 & 0.6753585 \\
\hline
\end{tabular}

Source: Encuesta de Estructura Salarial (INE, 2017) 